\title{
On the Scaling Property of Power Grids
}

\author{
Zhifang Wang, Seyyed Hamid Elyas \\ Electrical and Computer Engineering \\ Virginia Commonwealth University \\ Richmond VA, USA \\ \{zfwang, elyassh\}@vcu.edu
}

\begin{abstract}
Compared with other natural or man-made networks, electric power grid assumes distinct "electric" topology with special small-world properties and electrical parameter settings. In this paper we study the scaling property of power grid in terms of both topology measures and electric parameters, with a number of realistic power grid test cases of different size. The examined measures and parameters include average node degree, average path length, algebraic connectivity, the bus type entropy that characterize relative locations of generation and load buses, generation capacity, total demand, and transmission capacity. Interpreting and testing the scaling property of power grid will help us better understand the intrinsic characteristics of electric energy delivery network of this critical infrastructure; and enable the development of an appropriate synthetic modeling that could be utilized to generate power grid test cases with accurate grid topology and electric parameters.
\end{abstract}

Index Terms-Electric Power System Modeling, Scaling Property, Random Topology Grid.

\section{INTRODUCTION}

For many countries their electric power system is one of the most critical infrastructures whose reliable and efficient operation is necessary for the essential functioning of their society [1]. In order to develop, test, and validate new tools, techniques, and algorithms for grid enhancement power researchers need a large volume of realistic grid data. However, only very few and limited test cases and real-world power grid datasets such as [2] and [3] are publicly and freely available, due to various security concerns.

In order to address the urgent need of grid data for testing new approaches and designs, many synthetic models have been developed in the past, such as a tree-topology power grid model in references [4] and [5] to study power grid robustness and cascading failure blackouts, and a ring-structured power grid model developed in [6] to examine disturbance propagation. A small-world graph model for power grids was first introduced by Watts and Strogatz in [7]. A randomwalk grid model with Poisson or Uniform bus locations was proposed in [8]. Reference [9] also used a small-world graph model to study the spreading mechanism of chain failures in a large-scale grid. All these models provide useful perspectives

This paper was prepared as a result of work sponsored by the U.S. Department of Energy (DoE) partially through the Consortium for Electric Reliability Technology Solutions (CERTS) Program, and partially sponsored by the DoE ARPAE award DE-AR0000714. of power grid characteristics but fail to accurately or fully represent a realistic power system, especially its distinct sparse connectivity and scaling property versus the grid size. Besides, power grid networks are much more than a graph topology. In order to facilitate numerical simulations for grid controls and operations, one also needs to include realistic electrical parameter settings such as line impedances, generation and load settings, and transmission capacities. Our previous work [10] proposed a random-topology power grid model, called RT-nestedSmallWorld, which could be used to produce any needed number of power grid test cases with scalable network size featuring the same kind of small-world "electric" topology of real-world power transmission network. References [11] and [12] represent the first works that incorporate spatial information into synthetic power grid modeling. Reference [13] provides a survey of recent scientific studies that uses complex network analysis to examine the properties of power grid infrastructures .

This paper expands our past work on random topology grid modeling with a study on the scaling property of power grid in terms of both topology measures and electric parameters, with all the available realistic power grid test cases of different sizes. The examined measures and parameters include average node degree, average path length, algebraic connectivity, the bus type entropy that characterize relative locations of generation and load buses, generation capacity, total demand, and transmission capacities. Interpreting and testing the scaling property of power grids will help us better understand the intrinsic characteristics of this critical infrastructure of electric energy delivery system; and enable the development of an appropriate synthetic modeling that could be utilized to generate power grid test cases of scalable network size and with accurate grid topology and electric parameters.

A numerical measure called "Bus Type Entropy" in [14] is re-examined and revised in this paper to characterize the correlated assignment of generation, load, and connection buses in a power grid, based on the IEEE test cases and some newly obtained realistic grid data such as the ERCOT, WECC, and PEGASE systems. The revised the entropy definition has better statistical property and improved numerical stability. We can then derive an approximate scaling function of bus type assignment versus network size.

The rest of the paper is organized as follows. Section II introduce the system model and some related definitions. Section III examines some special scaling property of the 
connecting topology of a power grid in terms of average connectivity, average path length in hops, and algebraic connectivity. Section IV provides a revised definition of bus type entropy that characterized the correlated assignments of generation, load, and connection buses; and then derive an approximate scaling function of the bus type entropy versus network size. Section V presents some results on the statistics of generation capacity, load settings, and transmission capacity and their corresponding scaling property. Finally Section VI concludes the paper.

\section{SySTEM MODEL}

In order to describe the connecting topology of an undirected graph network with with $n$ vertices (nodes) and $m$ edges (branches) a Laplacian matrix can be used:

$$
L=A^{T} A,
$$

where $A:=\left(A_{l, k}\right)_{m \times n}$ is the branch-node incidence matrix, arbitrarily oriented and defined as: $A_{l, i}=1 ; A_{l, j}=-1$, if the $l^{\text {th }}$ branch is from node $i$ to node $j$ and $A_{l, k}=0, k \neq i, j$.

On the other hand, to describe the power-transmission topology of a grid which is more than a graph network, one shall utilize an admittance matrix $Y_{n \times n}$ defined as:

$$
Y=A^{T} \Lambda^{-1}\left(z_{l}\right) A,
$$

where $\Lambda^{-1}(\cdot)$ denotes the diagonal inverse matrix with a specified vector and $z_{l}$ the vector of branch impedances in a grid. Comparing equations (2) with (1), one may find that the admittance matrix of a power grid can be viewed as a complex weighted Laplacian. Obviously, the definition of (2) neglects the shunts in a grid and can be corrected by only revising its diagonal entries. However, ignoring the shunts may not cause big errors in some grid analysis, especially those only involving real power transmission such as the $D C$ power flow approximation [1], which is a widely used standard approach in optimizing flow dispatch and for assessing line overloads .

The flow distribution in a power grid follows Kirchhoff's voltage and current law and Ohm's law. Therefore the grid network constraints can be derived as follows:

$$
\begin{aligned}
& P(t)=B^{\prime}(t) \theta(t), \\
& F(t)=\Lambda\left(y_{l}\right) A \theta(t),
\end{aligned}
$$

where

$$
P(t)=\left[P_{G}(t),-P_{L}(t), P_{C}\right]^{T}
$$

represents the vector of net injected real power from generation, load and connection buses. Obviously the power injection from a connection bus equals zero, i.e., $P_{C}=0$. Note that with the definition of injected power (4), we assume that buses in a grid have been reordered so that the generation, load, and connection buses could be grouped as listed. Clearly the location of generators and loads plays a vital role in grid operation. $\theta(t)$ is the vector of phase angles, and $F(t)$ the vector of real-power delivered along the branches. The matrix $B^{\prime}(t)$ is defined as

$$
B^{\prime}(t)=A^{T} \Lambda\left(y_{l}\right) A,
$$

where $y_{l}=1 / x_{l}$ with $x_{l}$ the branch series reactance and $\Lambda\left(y_{l}\right)$ represents a diagonal matrix with entries of $\left\{y_{l}, \quad l=\right.$ $1,2, \cdots, m\}$. Alternatively we can have $B^{\prime}=-\operatorname{Img}(Y)$ where the shunts of the grid neglected in the admittance matrix.

Besides the network constraints, grid operation also needs to account for the constraints of generation capacity, load settings, and transmission capacity, such as

$$
\begin{gathered}
P_{G}^{\min } \leq P_{G} \leq P_{G}^{\max }, \\
P_{L}^{\min } \leq P_{L} \leq P_{L}^{\max }, \\
-F^{\max } \leq F \leq F^{\max } .
\end{gathered}
$$

In the following sections of the paper, we will examine the scaling property of power grid in terms of both topology measures and electric parameters, with some available realistic power grid test cases of different network size.

\section{The Scaling Property of GRid Connecting TOPOLOGY}

As mentioned in Section II, the connecting topology of a power grid can be fully described by its admittance matrix which contains both graph topology and electric parameters. Compared with the topology of other natural or man-made networks, power grid topology is very different in many ways [7]-[17], such as the salient small-world properties characterized by shorter average path length and higher clustering coefficients than those of an Erdös-Rényi random graphs [18] with the same network size and total number of links. The average node degree of a typical power grid does not scale with the network size but remains within a very strict range. Besides, the node degrees approximates a statistical distribution of a truncated geometric random variable with some mixture of an irregular discrete. The algebraic connectivity of a grid also exhibits some special scaling property [10]. Another important property of power grid is its heavy-tailed distribution of line impedances, which is well-fitted by a clipped double-ParetologNormal (dPIN) distribution [19].

The node degree of bus $i$ in a grid equals the total number of branches it connects and can be obtained from the $i$ th diagonal entry of the Laplacian matrix, i.e., $k_{i}=L(i, i)$. Then the average nodal degree of the grid is

$$
\langle k\rangle=\frac{1}{n} \sum_{i=1}^{n} L(i, i) .
$$

Given the connecting topology of a grid, we can run the Dijkstra's algorithm to calculate the shortest path length measured in hops between any two buses $i$ and $j$, i.e., $l_{i j}$. Then the average shortest path length of a grid is

$$
\langle l\rangle=\frac{2 \sum_{i, j} l_{i j}}{n(n-1)} .
$$

Another important topology measure is the second smallest eigenvalue of the Laplacian matrix, $\lambda_{2}(L)$, called the algebraic connectivity, with

$$
\left[\lambda_{1}, \lambda_{2}, \cdots, \lambda_{n}\right]=\operatorname{Eigen}(L) .
$$


As a fact the smallest eigenvalue of the Laplacian is always zero, i.e., $\lambda_{1}(L) \equiv 0$ and the number of times that 0 appears as an eigenvalue in the Laplacian is the total number of islanded components in the network. $\lambda_{2}(L)$ reflects the overall connectivity of a network and how fast information data can be broadcast across it. The eigenvalue $\lambda_{2}(L)$ is greater than 0 if and only if network is a connected graph. If the algebraic connectivity $\lambda_{2}(L)$ is close to zero, the network is close to being disconnected. Otherwise, if $\lambda_{2}(L) / n$ gets close to 1 , where $n$ is the network size, the grid tends to be a fully connected topology.

Table I presents some topology measures evaluated on the IEEE test cases and other real-world grids of different network size, where the PEGASE systems represent some European nation's grid at different levels of network reduction, the NYSIO system partially represents the New York interconnect in the US, and the RTE system is an equivalent of the French Grid. Fig. 1 shows the average node degree (9) of each grid system versus its network size. Obviously the average node degree of most sample grids does not scale but staying within a very stable region as $\langle k\rangle \in[2.0,3.5]$. Two exceptions are the PEGASE 89-bus system with $\langle k\rangle=4.72$ and the NYISO 2935-bus system with $\langle k\rangle=4.47$, which are unusually higher than the average node degree observed in the rest grid systems. This may be caused by the original grid's denser connecting topology or by the equalization approaches used in the network reduction.

Fig. 2 depicts the average path length in hops, as defined in (10), versus the network size for each sample grid in Table I, where the dashed line represents an approximate fitting curve of the observed scaling property as $\langle l\rangle \propto 6.205 \log n$. Note that for the purpose of simplicity, the logarithm in this section is with base 10 .

Fig. 3 plots the algebraic connectivity, as defined in (11), scaling curve of power grid versus network size. We can compares it with that of 1-Dimensional and 2-Dimensional lattices where 1D-lattice is a ring structured topology, with nodes connected with most adjacent neighbors on both sides. 2D-lattice is a regular two-dimension meshed grid with each

TABLE I. Topology Measures of Real-World Power Grids

\begin{tabular}{l|c|c|c|c}
\hline \hline & $(n, m)$ & $\langle k\rangle$ & $\langle l\rangle$ & $\lambda_{2}$ \\
\hline IEEE-24 & $(24,38)$ & 3.17 & 3.21 & 0.2132 \\
\hline IEEE-30 & $(30,41)$ & 2.73 & 3.31 & 0.2121 \\
\hline NE-39 & $(39,46)$ & 2.36 & 4.75 & 0.0762 \\
\hline IEEE-57 & $(57,80)$ & 2.81 & 4.95 & 0.0882 \\
\hline PEGASE-89 & $(89,210)$ & 4.72 & 3.87 & 0.1537 \\
\hline IEEE-118 & $(118,186)$ & 3.15 & 6.31 & $2.71 \mathrm{e}-2$ \\
\hline IEEE-300 & $(300,409)$ & 2.73 & 9.94 & $9.38 \mathrm{e}-3$ \\
\hline PEGASE-1354 & $(1354,1991)$ & 2.90 & 11.20 & $6.59 \mathrm{e}-3$ \\
\hline PEGASE-2869 & $(2869,4582)$ & 3.19 & 20.00 & $6.23 \mathrm{e}-4$ \\
\hline NYISO-2935 & $(2935,6567)$ & 4.47 & 16.43 & $1.42 \mathrm{e}-3$ \\
\hline RTE-6515 & $(6515,9037)$ & 2.77 & 14.95 & $1.93 \mathrm{e}-3$ \\
\hline \hline
\end{tabular}

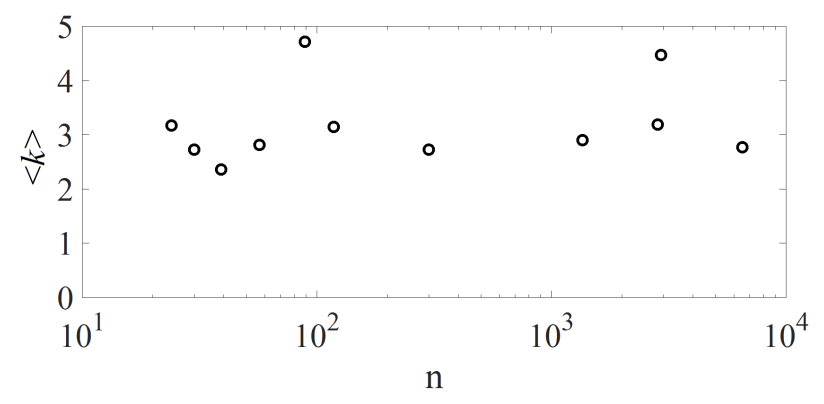

Figure 1. Average Node Degree versus the Network Size

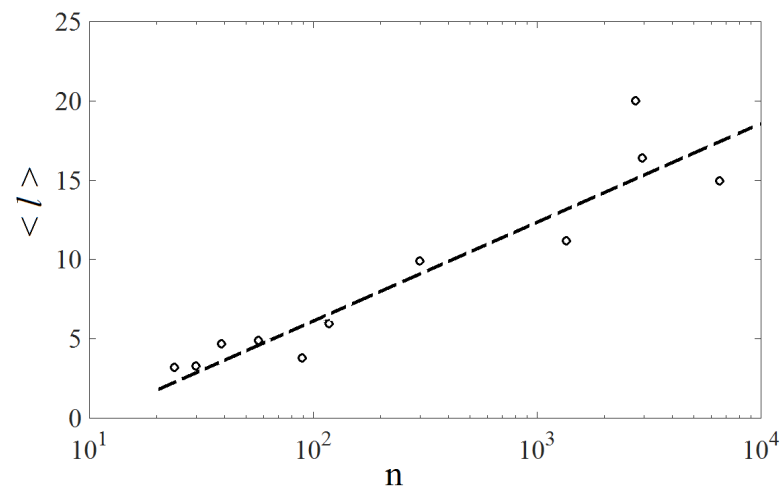

Figure 2. Average Path Length versus the Network Size

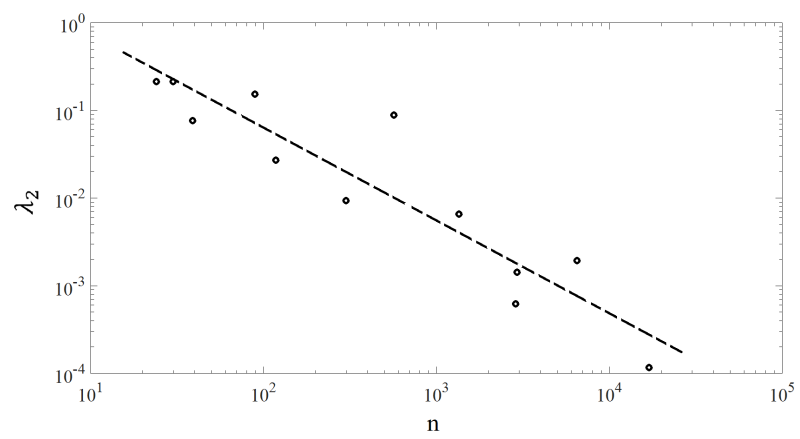

Figure 3. Algebraic Connectivity versus the Network Size

boundary side merging with the other side and each node connected to the most adjacent neighbors around it. For 1D lattice, its connectivity scales as $\lambda_{2}(L) \propto n^{-2}$; for 2-D lattice, its connectivity grows as $\lambda_{2}(L) \propto n^{-1}$; interestingly, for power grids, its connectivity grows as $\lambda_{2}(L) \propto n^{-1.041}$, lying between those of 1-D lattice and 2-D lattice.

Fig. 4 presents a scatter plot of the algebraic connectivity and the average path length of tested power grids, which exhibits strong correlation between the two measures. In fact there exists an approximate fitting function as $\log \left(\lambda_{2}\right) \propto$ $-0.1678\langle l\rangle$.

\section{The Scaling Property of Bus Type Assignment}

Now we examine the problem of how to site the three different types of buses $(\mathrm{G} / \mathrm{L} / \mathrm{C})$ in a given grid topology. In 


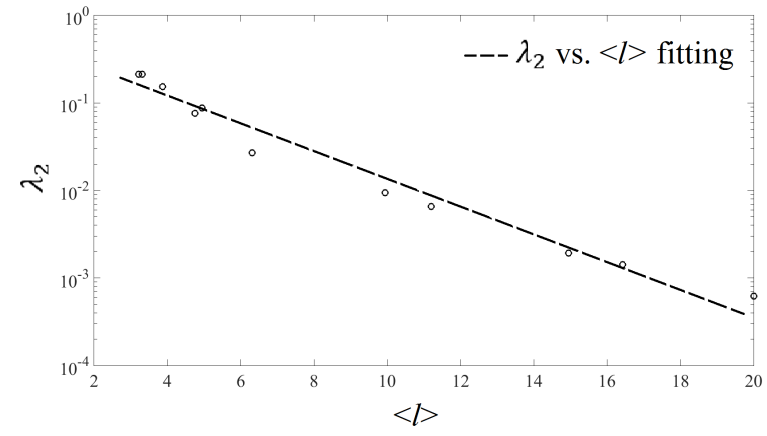

Figure 4. Algebraic Connectivity versus Average Path Length

a typical power grid we may find $20-40 \%$ of (G)eneration buses, $40-60 \%$ of (L)oad buses, and $10-20 \%$ of (C)onnection buses. It is noted that a small portion of buses in a grid may have both generation and load. For the purpose of bus type assignment study in this paper, we assume that the bus types of these buses will be decided based on the net injection power from the generation and load, $P=P_{G}-P_{L}$, under normal operation conditions. That is, a bus with positive net injection power is considered as a generation bus; a bus with negative net injection a load bus.

The topological location of generation, load, and connection buses in a grid can be fully described by an integer vector called bus type vector as defined later in this section. From [20] we know that a realict grid has correlated bus type assignment and non-trivial correlation exists between the bus types $(\mathrm{G} / \mathrm{L} / \mathrm{C})$ and other topology metrics such as the node degree distribution and the clustering coefficient. What is more, numerical simulations show that randomizing the original bus type assignment in a realistic grid will cause the grid to behave differently and lead to misleading results.

In order to study the scaling property of $(\mathrm{G} / \mathrm{L} / \mathrm{C})$ bus locations in a grid versue network size, we exploit a scalar measure called the Bus Type Entropy firsted introduced in [14]. The measure is defined with three variations and utilized to characterize the correlation of realistic bus type assignments. In this paper the entropy measures in [14] have been reexamined and revised so that an approximate scaling function of the correlated bus type assignment in realistic grids versus the network size can be appropriately derived, based on the IEEE test cases and some realistic grid data, such as the NYISO, the ERCOT, the WECC and the PEGASE systems.

\section{A. Bus Type Entropy $W(\mathbb{T})$}

We define the topological locations of generation, load, and connection buses in a $n$-bus, $m$-branch power transmission grid using a bus type vector $\mathbb{T}=\left[\mathbb{T}_{i}\right]_{n \times 1}$ with:

$$
\mathbb{T}_{i}= \begin{cases}1, & \text { if bus } i \text { is a }(\mathrm{G}) \text { eneration bus, } \\ 2, & \text { if bus } i \text { is a (L)oad bus, } \\ 3, & \text { if bus } i \text { is a (C)onnection bus, }\end{cases}
$$

where $i=1,2, \cdots, n$. If the grid is taken as an undirected graph, each transmission branch will assume one of the following six link types, i.e. $\{G G, G L, G C, L L, L C, C C\}$, fully determined by the bus types of the terminal buses in a given the grid topology. Therefore the link type vector of a grid can be defined as a function of $\mathbb{T}$ with:

$$
\mathbb{L}_{j} \in\{1,2,3,4,5,6\}, \quad j=1,2, \cdots, m,
$$

where the link type value of line $j$ is numbered according to the same order in above set.

Reference [14] proposed a scalar measure called Bus Type Entropy with three variations, denoted as $W_{1-3}(\mathbb{T})$, based on the bus type vector and the link type vector defined as above. With this measure we expect to distinguish a correlated bus type assignments $\left\{\mathbb{T}^{*}\right\}$, extracted from realistic grids, from the other randomized ones generated from permutation $\widetilde{\mathbb{T}}=\mathcal{P}\left(\mathbb{T}^{*}\right)$. By the central limit theorem, the randomized entropy values may assume a normal (Gaussian) distribution. With the extracted distribution parameters $(\mu, \sigma)$, an normalized distance can be defined to measure the difference between $\mathbb{T}^{*}$ and $\widetilde{\mathbb{T}}$ as:

$$
d_{W}\left(\mathbb{T}^{*}, \widetilde{\mathbb{T}}\right)=\frac{W\left(\mathbb{T}^{*}\right)-\mu}{\sigma}
$$

Obviously an accurate evaluation of the distance depends on how accurately the distribution parameters will be estimated from an empirical probability density function (PDF) of $W(\widetilde{\mathbb{T}})$ based on an appropriate entropy definition.

Our initial experiments on some synthetic and realistic grid systems in [14] detected a clearly shifting pattern of (14) versus the grid network size. Therefore in this paper, with more available grid data, we wish to study and derive an approximate scaling function of $d_{W}\left(\mathbb{T}^{*}, \widetilde{\mathbb{T}}\right)$ versus the network size $n$.

Using IEEE test cases of 30-300 buses and additional realistic grid data such as the NYISO, the ERCOT, and the WECC systems, we reexamine the entropy definitions in [14] and found that they are not very suitable for the desired study here because sometimes they may introduce segmented PDF curves, making difficult an accurate estimation of distribution parameters. Besides, the resulting entropy value seems to lack numerical stability, which is not ideal for the design of a searching algorithm we wish to develop later. Therefore in this paper we revise the definition of the bus type entropy as:

$$
W(\mathbb{T})=-\frac{\sum_{i=1}^{n} \log \left(r_{\mathbb{T}_{i}}\right)}{n}-\frac{\sum_{j=1}^{m} \log \left(R_{\mathbb{L}_{j}}\right)}{m},
$$

where $r_{\mathbb{T}_{i}}=\mathfrak{n}_{\mathbb{T}_{i}} / n$ represent the bus type ratio of bus $i$ and $R_{\mathbb{L}_{j}}=\mathfrak{m}_{\mathbb{L}_{j}} / m$ the corresponding link type ratio of the $j$ th line; and $\mathfrak{n}_{k}$ and $\mathfrak{m}_{k}$ representing the total number of buses and lines of different types in the grid that have some specified types respectively. Equivalently, the bus type entropy of (15) can also be written as:

$$
W(\mathbb{T})=-\Sigma_{k=1}^{3} r_{k} \log \left(r_{k}\right)-\Sigma_{k=1}^{6} R_{k} \log \left(R_{k}\right) .
$$

The revised entropy measure (16) is more like a typical "entropy" definition. The following examination verifies that entropy values calculated based on this definition will remain within a narrower range implying better numerical stability. 
Another advantage of this entropy definition is that all the empirical PDF derived for randomized bus type assignments will approximate a normal distribution and have not segmented patterns.

\section{B. Deriving a Scaling Function of $d_{W}(n)$}

Using the new definition of bus type entropy proposed in last subsection, we do the empirical probability analysis on the IEEE test cases and realistic grid data. Table II presents the evaluation results such as the fitting parameters $(\mu, \sigma)$ of the normal distribution of randomized bus type assignments, and the relative distance $d_{W}\left(\mathbb{T}^{*}, \widetilde{\mathbb{T}}\right)$, from which one may easily notice a shifting pattern of $d_{W}$ versus the network size.

Fig. 5 presents a sample empirical PDF evaluated for the NYISO 2935-bus system, with a sampling size of $k^{\max }=25,000$, in which the empirical PDF are depicted as bar plots and the fitting Normal distribution plotted as thicker blue line. The empirical probability distribution function (PDF) of a random variable $W$ can be generated through the normalized histogram analysis of $W(\widetilde{T})$ as

$$
f_{W}(x)=\frac{\Sigma_{k=1}^{k^{\max }} \delta_{\Delta}\left(W_{k}-x\right)}{k^{\max }},
$$

where $\delta_{\Delta}(\cdot)$ is a Dirac Delta function with a width of $\Delta$ :

$$
\delta_{\Delta}(x)= \begin{cases}\frac{1}{\Delta}, & |x| \leq \frac{\Delta}{2} \\ 0, & \text { otherewise. }\end{cases}
$$

Fig.5 implies that the "correlated" bus type assignment in a large realistic power grid such as the NYISO 2935-bus system stands out from those randomized bus type assignments, although implemented on the same connecting topology, with the help of an appropriately defined entropy measure (15). More discussion on the ability of the proposed entropy measure to characterize correlated bus type assignments in realistic grids can be found in [14] and [21].

With above results we apply a curving fitting method to $d_{W}\left(\mathbb{T}^{*}, \widetilde{\mathbb{T}}\right)$ and derive an approximate scaling function as:

$$
d_{W}(n)=\left\{\begin{array}{ll}
-1.39 \ln n+6.79, & \ln n \leq 8 \\
-1.25 \times 10^{-13}(\ln n)^{15.1}+0.43, & \ln n>8
\end{array},\right.
$$

TABLE II. The Normal Fitting PARAmeters of the EMPIRICAL PDF of $W(\widetilde{\mathbb{T}})$ AND THE RELATIVE Distance of $d_{W}$

\begin{tabular}{l|c|r|r}
\hline \hline & $(\mu, \sigma)$ & $W\left(\mathbb{T}^{*}\right)$ & $d_{W}\left(\mathbb{T}^{*}, \widetilde{T}\right)$ \\
\hline IEEE-30 & $(2.38,9.0 \mathrm{e}-2)$ & 2.49 & 1.22 \\
\hline IEEE-57 & $(2.31,5.8 \mathrm{e}-2)$ & 2.44 & 2.24 \\
\hline IEEE-118 & $(2.34,4.5 \mathrm{e}-2)$ & 2.35 & 0.22 \\
\hline IEEE-300 & $(2.57,2.6 \mathrm{e}-2)$ & 2.53 & -1.53 \\
\hline NYISO-2935 & $(2.75,7.3 \mathrm{e}-3)$ & 2.70 & -5.71 \\
\hline ERCOT-5633 & $(2.36,8.1 \mathrm{e}-3)$ & 2.23 & -16.25 \\
\hline WECC-16994 & $(2.72,3.4 \mathrm{e}-3)$ & 2.33 & -114.70 \\
\hline \hline
\end{tabular}

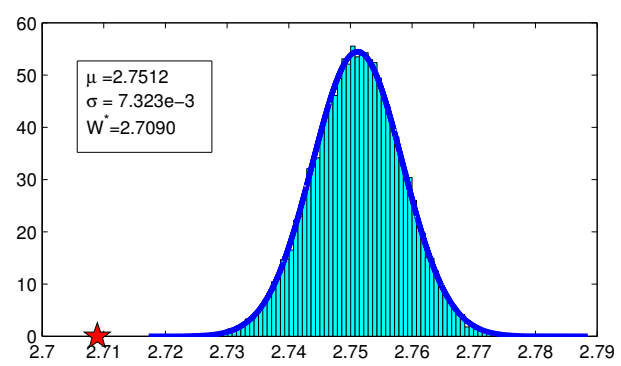

(a)

Figure 5. The Empirical PDF and the Normal Distribution Fitting for the Bus Type Entropy $W(\widetilde{\mathbb{T}})$ by Randomizing the Bus type Assignments in the NYISO 2935-bus System: the realistic-grid bus type entropy $W^{*}$ is marked by a red 'star'.

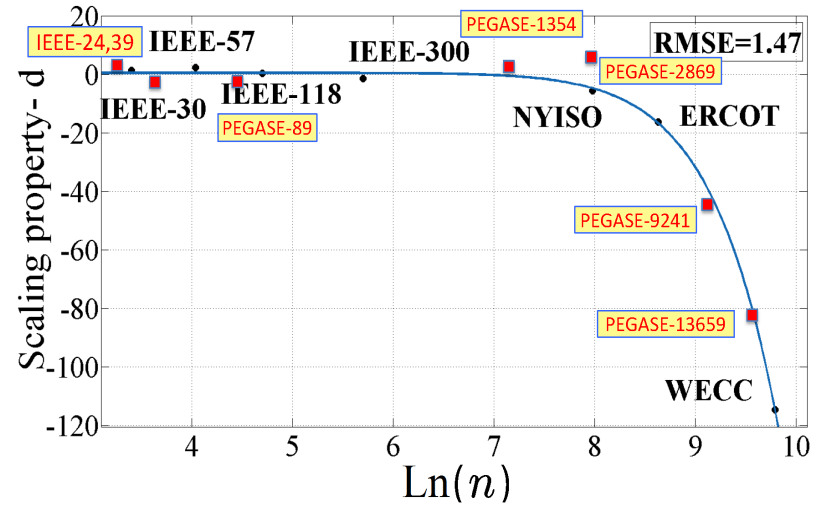

Figure 6. The fitting curve of the scaling function of the relative distance $d_{w}$ versus the network size $n$, where the data points marked as 'red squares' represent the evaluation results obtained from additional grid test cases for verification.

where the logarithm is natural logarithm with base e. Fig.6 shows the approximate scaling function of (19) with a rootmean-square error (RMSE) of 1.47, evaluated based on the numerical results of realistic grids as listed in Table II. In order to verify the accuracy of the derived approximate scaling function, we test on other realistic grid data such as the IEEE24, NE-39 buses systems and the PEGASE systems at different levels of network reduction, with the addtional evaluation results lised in Table III. The evaluation results obtained from additional grid test cases for verification are depicted as scattered data points marked by "red squares" in Fig.6, which seem to match very well with the previously derived scaling function, except one test case, i.e. the PEGASE-2869 system. The non-trivial deviation of this system from the scaling curve we think might come from an inaccurate network reduction which causes the relative location of generation and loads inconsistent with the original grid settings.

Therefore in our synthetic grid modeling, we will be able to utilize a scaling function as below to determine the target entropy value for the correlated bus type assignments $W^{*}(n)=W\left(\mathbb{T}^{*} \mid n\right)$ :

$$
W^{*}(n)=\mu+\sigma \cdot d_{W}(n)
$$


where $d_{W}(n)$ is calculated using (14), and $(\mu, \sigma)$ estimated from the empirical PDF of the entropy values of randomized bus type assignments in a given grid topology with specified bus type ratios.

\section{The Scaling Property of Other Electric PARAMETERS}

After studying the scaling property of the small-world connecting topology and determining the topological location of generation and loads in a grid, it would be ideal and same critical to further examine the scaling property of other electric parameters such as the generation capacities, load settings, and transmission capacity, just as indicated in Section II.

Table IV presents the evaluation results of total generation capacity, total demand, and total backbone transmission capacity of some realistic grids, with the corresponding definitions as:

$$
\begin{aligned}
P_{G, \max }^{\mathrm{tot}} & =\sum_{i \in G} P_{G, \max }^{i}, \\
P_{L}^{\mathrm{tot}} & =\sum_{i \in L} P_{L}^{i}, \\
F_{\max }^{\mathrm{tot}} & =\sum_{l \in \mathrm{BKB}} F_{\max }^{l} .
\end{aligned}
$$

Fig.7 plots the total generation capacity and total demand in the grid systems as listed in Table IV and the ratio between the two versus the network size. The scaling functions obtained from the curving fitting approach are given as follows:

$$
\begin{aligned}
& \log P_{G, \max }^{\mathrm{tot}}(n)=-0.21(\log n)^{2}+2.06(\log n)+0.66, \\
& \log P_{L}^{\mathrm{tot}}(n)=-0.20(\log n)^{2}+1.98(\log n)+0.58,
\end{aligned}
$$

where both $P^{t o t}(n)$ 's are measured in MW and the logarithm is with base 10 .

From Fig.7 we can see that when the network size is small (i.e. $n<300$ ), the total generation capacity and demand in a grid tend to grow as a power function, i.e., $P^{\text {tot }} \propto n^{2.0}$. However, as the network size becomes larger, the scaling curves begin to bend down and grow slower than that power function. However the ratio of the two, $r_{G / L}=P_{G, \max }^{\text {tot }} / P_{L}^{\text {tot }}$, tends to slowly drop down from above 1.50 and draw closer to 1.00 as the network size increases.

TABLE III. AdDitional EVAluation Results of the Relative DisTANCE OF $d_{W}\left(\mathbb{T}^{*}, \widetilde{T}\right)$

\begin{tabular}{l|c|c|r}
\hline \hline & $(\mu, \sigma)$ & $W\left(\mathbb{T}^{*}\right)$ & $d_{W}\left(\mathbb{T}^{*}, \widetilde{\mathbb{T}}\right)$ \\
\hline IEEE-24 & $(2.5,8.4 \mathrm{e}-2)$ & 2.61 & 1.30 \\
\hline NE-39 & $(2.65,6.4 \mathrm{e}-2)$ & 2.51 & -2.16 \\
\hline PEGASE-89 & $(2.51,6.5 \mathrm{e}-2)$ & 2.38 & -2.01 \\
\hline PEGASE-1354 & $(2.66,1.0 \mathrm{e}-2)$ & 2.64 & 1.90 \\
\hline PEGASE-2869 & $(2.62,7.5 \mathrm{e}-3)$ & 2.65 & 4.01 \\
\hline PEGASE-9241 & $(2.84,6.0 \mathrm{e}-3)$ & 2.58 & -43.33 \\
\hline PEGASE-13659 & $(2.8,3.0 \mathrm{e}-3)$ & 2.56 & -80.70 \\
\hline \hline
\end{tabular}

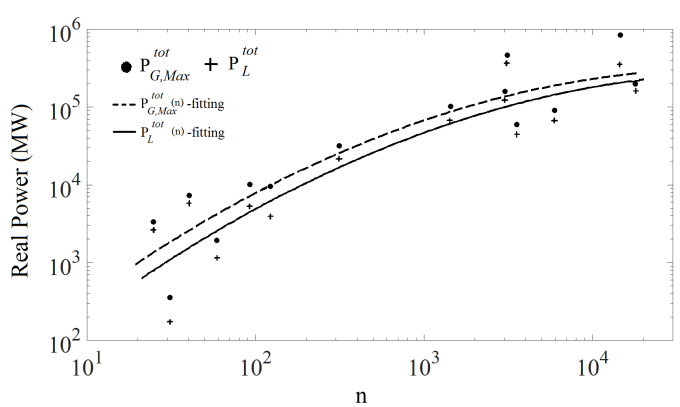

(a)

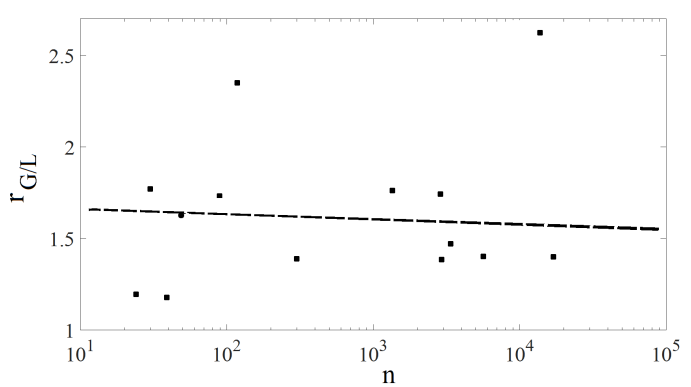

(b)

Figure 7. The scaling property of the total generation capacity and demand in realistic power grids:(a) the total generation marked by 'black squares' and total demand marked by ' +' ; (b) the ratio of the total generation to the total demand.

Our initial study on the statistical distribution of generation capacity and demand within a power grid based on some realistic grid data such as the PEGASE, the WECC, and the NYISO systems, shows that the generation capacity and load settings approximately follow an exponential distribution, as shown in Fig.8-10. It is interesting to note that in the PEGASE 13659-bus system, about $99.9 \%$ of the generators have capacities following an exponential distribution except

TABlE IV. Total Generation CAPacity, Demand, And Total BACKBONE TRANSMISSION CAPACITY IN SOME REALISTIC GRIDS

\begin{tabular}{l|c|c|c|c}
\hline \hline & $P_{G, \max }^{\text {tot }}(\mathrm{MW})$ & $P_{L}^{\text {tot }}(\mathrm{MW})$ & $r_{G / L}$ & $F_{\max }^{\text {tot }}(\mathrm{MW})$ \\
\hline IEEE-24 & $3.41 \mathrm{e} 3$ & $2.85 \mathrm{e} 3$ & 1.19 & $1.87 \mathrm{e} 4$ \\
\hline IEEE-30 & $3.35 \mathrm{e} 2$ & $1.89 \mathrm{e} 2$ & 1.77 & $1.95 \mathrm{e} 3$ \\
\hline NE-39 & $7.37 \mathrm{e} 3$ & $6.25 \mathrm{e} 3$ & 1.18 & $3.37 \mathrm{e} 4$ \\
\hline IEEE-57 & $1.98 \mathrm{e} 3$ & $1.25 \mathrm{e} 3$ & 1.58 & - \\
\hline PEGASE-89 & $9.92 \mathrm{e} 3$ & $5.73 \mathrm{e} 3$ & 1.73 & $9.59 \mathrm{e} 4$ \\
\hline IEEE-118 & $9.97 \mathrm{e} 3$ & $4.24 \mathrm{e} 3$ & 2.35 & - \\
\hline IEEE-300 & $3.27 \mathrm{e} 4$ & $2.35 \mathrm{e} 4$ & 1.39 & - \\
\hline PEGASE-1354 & $1.29 \mathrm{e} 5$ & $7.31 \mathrm{e} 4$ & 1.76 & $8.99 \mathrm{e} 5$ \\
\hline PEGASE-2869 & $2.31 \mathrm{e} 5$ & $1.32 \mathrm{e} 5$ & 1.74 & $1.93 \mathrm{e} 6$ \\
\hline NYISO-2935 & $5.47 \mathrm{e} 5$ & $3.95 \mathrm{e} 5$ & 1.38 & $2.65 \mathrm{e} 5$ \\
\hline Polish-3375 & $7.11 \mathrm{e} 4$ & $4.84 \mathrm{e} 4$ & 1.47 & $1.37 \mathrm{e} 6$ \\
\hline ERCOT-5633 & $1.02 \mathrm{e} 5$ & $7.28 \mathrm{e} 4$ & 1.40 & $1.84 \mathrm{e} 6$ \\
\hline PEGASE-13659 & $9.81 \mathrm{e} 5$ & $3.81 \mathrm{e} 5$ & 2.57 & $2.27 \mathrm{e} 6$ \\
\hline WECC-16994 & $2.46 \mathrm{e} 5$ & $1.74 \mathrm{e} 5$ & 1.41 & $4.49 \mathrm{e} 6$ \\
\hline \hline
\end{tabular}




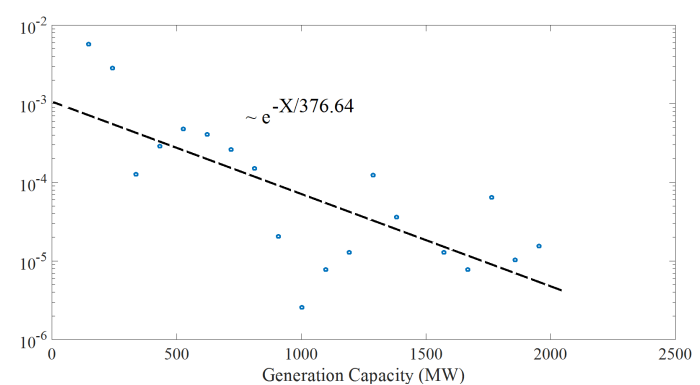

(a)

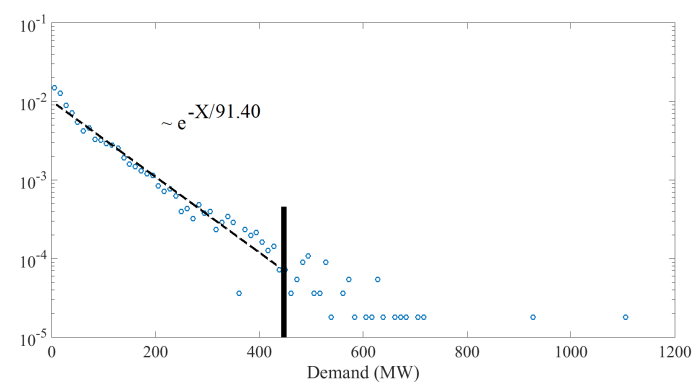

(b)

Figure 8. The empirical PDF of generator capacities and demands in the PEGASE-13659 bus system

$0.1 \%$ with very large capacities falling out of the normal range. In the WECC 16994-bus system and the NYISO 2935-bus systems, approximately $99 \%$ of the generation capacities (and the loads as well) follow an exponential distribution while only $1 \%$ with extremely large capacities (or demands) falling out of the expected normal range. We will continue the study on this aspect to determine the cause of observed distribution exceptions, which may either come from an inherent heavytailed distribution or only result from boundary equivalization in a network reduction modeling. Our study also shows that there exists correlations between the total number of branches connecting a bus (that is, its node degree) and the generation or load attached to the bus.

Fig.11 shows the scaling property of total backbone transmission capacity in a grid. The fitting curve is depicted as a dashed line for the scaling function of $F_{\max }^{\text {tot }}(n)$. The straight line in the log-log plot implies that the total transmission capacity of the grid backbone branches grows as a power function, $F_{\max }^{\text {tot }}(n) \propto n^{0.9059}$.

\section{CONCLUSIONS AND Future WORK}

This paper expands our past work on random topology grid modeling with a study on the scaling property of power grid in terms of both topology measures and electric parameters, with all the available realistic power grid test cases of different sizes. The examined measures and parameters include average node degree, average path length, algebraic connectivity, the bus type entropy that characterize relative locations of generation and load buses, generation capacity, total demand, and transmission capacities. Interpreting and testing the scaling

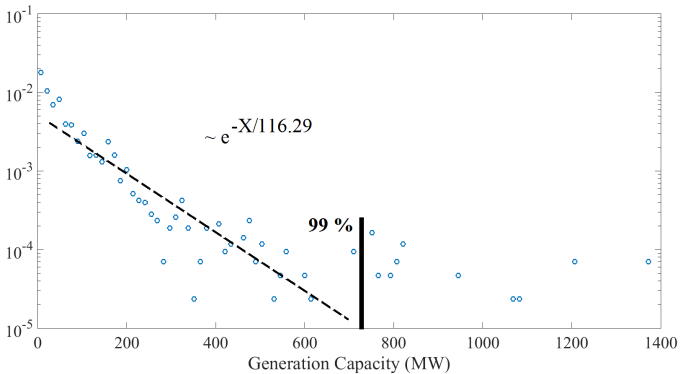

(a)

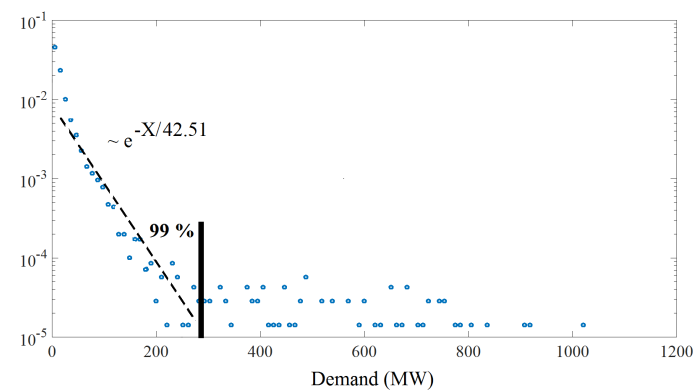

(b)

Figure 9. The empirical PDF of generator capacities and demands in the WECC-16994 bus system

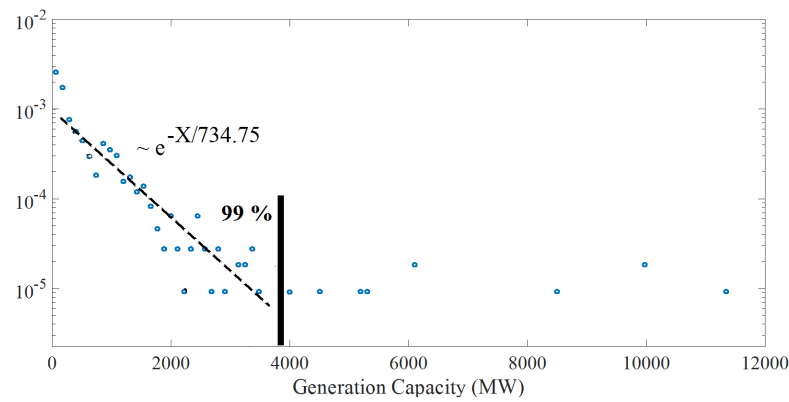

(a)

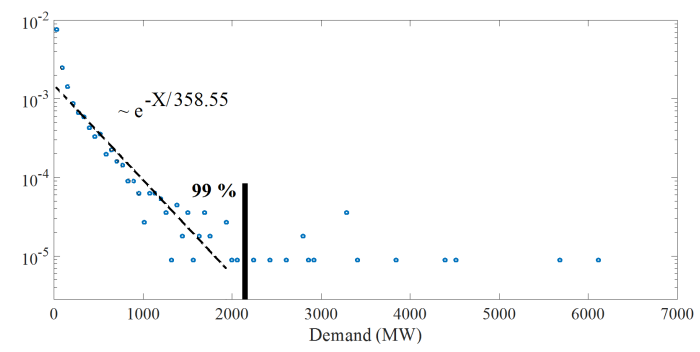

(b)

Figure 10. The empirical PDF of generator capacities and demands in power grids in the NYISO-2935 bus system

property of power grid help us better understand the intrinsic characteristics of this critical infrastructure of electric energy delivery; and enable the development of an appropriate syn- 


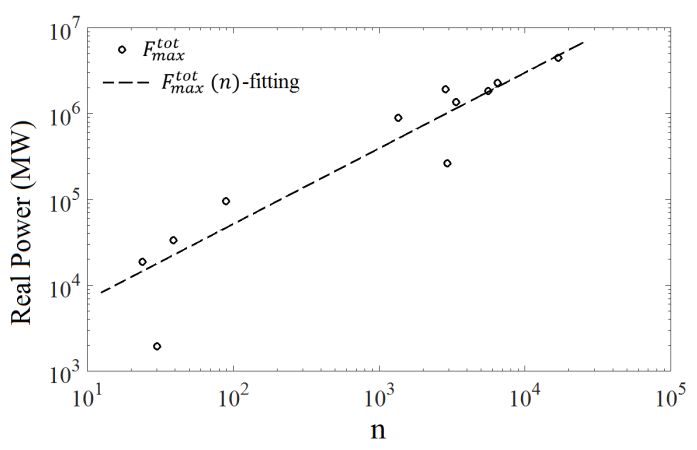

Figure 11. The scaling property of the total backbone transmission capacity in realistic power grids.

thetic modeling that could be utilized to generate power grid test cases of scalable network size and with accurate grid topology and electric parameters.

We have improved and redefined a novel topology measure, called the Bus Type Entropy, to characterize the bus type assignment of realistic power grids. The newly proposed measure has better numerical stability since it follows more strict entropy definition therefore it will simplify our analysis of the scaling property of the entropy value versus the network size. We examine the performance of the proposed measure on the IEEE test cases, the NYISO, the ERCOT, and the WECC systems based on the empirical probability density function analysis. Numerical results obtained from both systems verify the effectiveness of the proposed measure to characterize the correlated bus type assignment of a real-world power grid and help us determine an scaling function of bus type assignment versus network size. The derived approximate scaling property based on the entropy definition has been further verified with additional experiment results obtained from IEEE 24, NE-39 buses systems and some PEGASE systems with different levels of network reduction.

This paper also presents our initial study on the scaling property of electric parameters such as total generation capacity, total demand, and backbone transmission capacity. It shows that when the network size is small, the total generation capacity and demand in a grid tend to grow as a power function, $P^{\text {tot }} \propto n^{2.0}$. However, as the network size becomes larger, the scaling curves begin to bend down and grow slower than that power function. However the ratio of the two tends to slowly drop down from above 1.50 getting closer to 1.00 as the network size increases. The total backbone transmission capacity tends to grow as a power function of network size, $F_{\max }^{\text {tot }}(n) \propto n^{0.9059}$.

Our study on the statistical distribution of generation capacity and demand within a power grid based on some realistic grid data such as the PEGASE, the WECC, and the NYISO systems, shows that the generation capacity and load settings approximately follow an exponential distribution. It is found that most $(>99 \%)$ generators (and loads as well) in these two power grids have the capacities (or demands) assuming an exponential distribution with a very small portion of exceptions with extremely large generation capacity (or demands).

In the future further experiments and verification on other realistic power grid data and based on grid vulnerability analysis will be performed. The scaling properties obtained from the realistic grids will be incorporated into our synthetic grid modeling.

\section{REFERENCES}

[1] A. Wood and B. Wollenberg, Power System Generation, Operation and Control. New York: Wiley, 1984

[2] Power systems test case archive [Online]. Available: http://www.ee.washington.edu/research/pstca/.

[3] MATPOWER test cases [Online]. Available: http://www.pserc.cornell.edu/matpower/

[4] B. A. Carreras, V. E. Lynch, I. Dobson, and D. E. Newman, "Critical points and transitions in an electric power transmission model for cascading failure blackouts," Chaos, volume 12(4), 2002, pp.985-994.

[5] M. Rosas-Casals, S. Valverde, and R. V. Sol, "Topological Vulnerability of the European Power Grid under Errors and Attacks", Int. J. Bifurcation Chaos 17, pp.2465-2475, 2007.

[6] M. Parashar, J. S. Thorp, "Continuum modeling of electromechanical dynamics in large-scale power systems," IEEE Trans. on Circuits and Systems, volume 51(9), 2004, pp.1848-1858.

[7] D. J. Watts, S. H. Strogatz, "Collective dynamics of 'Small-World' networks," Nature, volume 393, 1998, pp.393-440.

[8] Z. Wang, R. J. Thomas, A. Scaglione, "Generating random topology power grids," Proc. 41st Annual Hawaii International Conference on System Sciences (HICSS-41), volume 41, p. 183, Big Island, Jan 2008.

[9] L. Fu, W. Huang, S. Xiao; Y. Li; S. Guo, "Vulnerability Assessment for Power Grid Based on Small-world Topological Model," Power and Energy Engineering Conference (APPEEC), 2010 Asia-Pacific , pp.1-4, 28-31 March 2010.

[10] Z. Wang, A. Scaglione, and R. J. Thomas, "Generating Statistically Correct Random Topologies for Testing Smart Grid Communication and Control Networks", IEEE Transactions on Smart Grid, volume 1(1):2839, 2010.

[11] S. Soltan and G. Zussman,"Generation of synthetic spatially embedded power grid networks", arXiv:1508.04447, 2015.

[12] K. M. Gegner, A. B. Birchfield, T. Xu, K. S. Shetye, and T. J. Overbye, "A methodology for the creation of geographically realistic synthetic power flow models", 2016 IEEE Power and Energy Conference at Illinois (PECI), pp. 16, 2016.

[13] G. A. Pagani, M. Aiello,"The power grid as a complex network: a survey", Physica A: Statistical Mechanics and its Applications, volume 392, issue 11, pp 2688-2700, 2013.

[14] Z. Wang, S.H. Elyas, and R. J. Thomas, "A novel measure to characterize bus type assignments of realistic power grids", PowerTech 2015, Eindhoven, Netherlands, June 2015.

[15] P. Hines, S. Blumsack, E. Cotilla Sanchez, and C. Barrows, "The Topological and Electrical Structure of Power Grids," Proc. 2010 43rd Hawaii International Conference on System Sciences (HICSS-43), Jan 2010.

[16] E. Cotilla Sanchez, P. Hines, C. Barrows, and S. Blumsack, "Comparing the Topological and Electrical Structure of the North American Electric Power Infrastructure," IEEE Systems Journal, volume 6, no. 4, pp.616626, 2012.

[17] R. Albert and A. Barabási, "Statistical mechanics of complex networks," Reviews of Modern Physics, volume 74(1), 2002, pp.47-97.

[18] P. Erdös, and A. Rényi, "On random graphs. I.." Publicationes Mathematicae, volume 6, 1959, pp.290-297.

[19] W. J. Reed, and M. Jorgensen, "The double pareto-lognormal distribution: a new parametric model for size distributions," Communications in statistics, Theory and methods, volume 33(8), 2004, pp.1733-1753.

[20] Z. Wang, R. J. Thomas, "On Bus Type Assignments in Random Topology Power Grid Models", HICSS'48, Jan 2015.

[21] S. H. Elyas, Z. Wang "A Multi-objective Optimization Algorithm for Bus Type Assignments in Random Topology Power Grid Model”, HICSS'49, 2016. 